\title{
Review paper on performance analysis of AODV, DSDV, OLSR on the basis of packet delivery
}

\author{
Ramandeep Kaur ( M. Tech Student), Chandan Sharma (Ph.D. Candidate), \\ Shri Guru Granth Sahib World University, Fatehgarh Sahib, India \\ Punjab Technical University, Jalandhar, India(pb.)
}

\begin{abstract}
The field of Adhoc Network has gained an important part of the interest of researchers and become very popular in last few years. Adhoc network can operate without fixed infrastructure and can survive rapid changes in the network topology. They can be studied formally as graphs in which the set of edges varies in time. In this paper, we analyse the performance of three protocols AODV, DSDV OLSR. The performance of proposed networks are evaluated in terms of packet delivery ratio with the help of NS-3 simulator. In this paper, OLSR shows better performance over the other two protocols, that is DSDV and AODV.
\end{abstract}

Keywords: AODV, DSDV, OLSR and NS-3

\section{Introduction}

A wireless ad hoc network is a decentralized type of wireless network. The network is ad hoc because it does not rely on a preexisting infrastructure, such as routers in wired networks or access points in managed (infrastructure) wireless networks [2]. Instead, each node participates in routing by forwarding data for other nodes, and so the determination of which nodes forward data is made dynamically based on the network connectivity. In addition to the classic routing, ad hoc networks can use flooding for forwarding the data.

An ad hoc network typically refers to any set of networks where all devices have equal status on a network and are free to associate with any other ad hoc network devices in link range. Very often, ad hoc network refers to a mode of operation of IEEE 802.11 wireless networks. The ad hoc routing protocols can be divided into two classes: table-driven and on-demand routing, on the basis of when and how the routes are discovered. In table-driven routing protocols, consistent and up-to date routing information to all nodes is maintained at each node, whereas in on-demand routing the routes are created only when desired by the source host. We discuss current table-driven protocols as well as on-demand protocols.

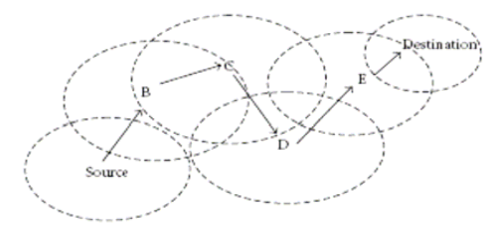

Fig 1. Adhoc networking model

In section 2, we review the mostly used wireless ad hoc protocols. In Section 3, we present the performance metrics of our simulation. Section 4 described the simulation environment, Section 5 performance comparison of the protocols. We draw our conclusions in Section 6. Future work in section 7.

\section{Adhoc Routing Protocols}

Routing protocols in MANETs are classified into three different categories according to their functionality
A. Reactive protocols
B. Proactive protocols
C. Hybrid protocols 


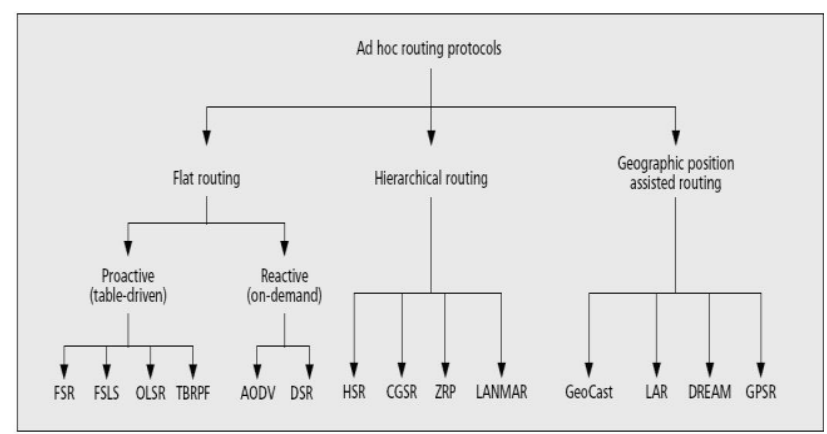

Fig. 2 Classification of routing protocols

\section{A. Reactive Protocols}

Reactive protocols are also known as On-demand driven reactive protocols. These Protocols do not initiate route discovery by themselves, until or unless a source node request to find a route. That's why these protocols are called reactive protocols. These protocols setup routes when demanded [4], [3]. When a node wants to communicate with another node in the network, and the source node does not have a route to the node it wants to communicate with, reactive routing protocols will establish a route for the source to destination node. Normally reactive protocols

- Don't find route until demanded

- Uses flooding technique to propagate the query, to find the destination - On-Demandll.

- Do not consume bandwidth for sending information.

- They consume bandwidth only, when the node start transmitting the data to the destination node.

Some of the most used on demand routing protocols are DSR [5], [6], AODV [4], [12] and Admission Control enabled On demand Routing Protocol( ACOR).

\section{B. Proactive Protocols}

Proactive routing protocols work as the other way around as compared to Reactive routing protocols. These protocols constantly maintain update-to-date topology of the network. Every node in the network knows about the other node in advance, in other words the whole network is known to all the nodes making that network. All the routing information is usually kept in tables. Whenever there is a change in the network topology, these tables are updated according to the change. The nodes exchange topology information with each other; they can have route information any time when they needed. Some of the existing proactive routing protocols are DSDV [7], OLSR [8] and Wireless Routing Protocol (WRP).

\section{Hybrid Protocols}

Hybrid protocols exploit the strengths of both reactive and proactive protocols, and combine them together to get better results. The network is divided into zones, and use different protocols in two different zones i.e. one protocol is used within zone, and the other protocol is used between them. Zone Routing Protocol (ZRP) is the example of Hybrid Routing Protocol. ZRP uses proactive mechanism for route establishment within the nodes neighbourhood, and for communication amongst the neighbourhood it takes the advantage of reactive protocols. These local neighbourhoods are known as zones, and the protocol is named for the same reason as zone routing protocol. Each zone can have different size and each node may be within multiple overlapping zones. The size of zone is given by radius of length $\mathrm{P}$, where $\mathrm{P}$ is number of hops to the perimeter of the zone [9]. Some of the existing hybrid protocols are ZRP [10], TORA [11] and Hazed Sighted Link State Routing Protocol (HSLS).

Dsdv- Destination Sequenced Distance Vector (DSDV) is a Proactive routing protocol that solves the major problem associated with the Distance Vector routing of wired. The DSDV protocol requires each mobile station to advertise, to each of its current neighbours, its own routing table (for instance, by broadcasting its entries). The entries in this list may change fairly dynamically over time, so the advertisement must be made often enough to ensure that every mobile computer can almost always locate every other mobile computer. In addition, each mobile computer agrees to relay data packets to other computers upon request. At all instants, the DSDV protocol guarantees loop-free paths to each destination [1].

AODV- AODV offers low network utilization and uses destination sequence number to ensure loop freedom. It is a reactive protocol implying that it requests a route when needed and it does not maintain routes for those nodes that do not actively 
participate in a communication. An important feature of AODV is that it uses a destination sequence number, which corresponds to a destination node that was requested by a routing sender node. The destination itself provides the number along with the route it has to take to reach from the request sender node up to the destination. If there are multiple routes from a request sender to a destination, the sender takes the route with a higher sequence number. This ensures that the ad hoc network protocol remains loop-free [1].

OLSR- Optimized Link State Routing (OLSR) is a link state routing protocol. OLSR is an adoption of conventional routing protocols to work in an ad hoc network on top of IMEP.

The novel attribute of OLSR is its ability to track and use multipoint relays. The idea of multipoint relays is to minimize the flooding of broadcast messages in the network by reducing/optimizing duplicate retransmissio-

-ns in the same region. Each node in the network selects a set of nodes in its neighbourhood that will retransmit its broadcast packets. This set of selected neighbor nodes is called the multipoint relays of that node. Each node selects its multipoint relay set in a manner to cover all the nodes that are two hops away from it. The neighbors that are not in the multipoint relay set still receive and process broadcast packets, but do not retransmit them [13].

\section{Performance Metrics}

We evaluated key performance metrics for three different applications using AODV, DSDV, OLSR protocols. The parameters used for wifi performance evalution is packet delivery ratio (packet send and received).

\section{Simulation}

Our protocol evaluation are based on the simulation using NS-3 simulator. The simulation runs for 600 simulated seconds, of which the first 50 nodes are used for start up time. The number of nodes are 50 . Nodes move according to Radom Waypoint Mobility Model with speed of $20 \mathrm{~m} / \mathrm{s}$ and no pause time within a $300 * 1500$ region. The wifi is in adhoc mode with a $2 \mathrm{Mb} / \mathrm{s}$ rate (802.11b) and a friss loss model. The transmit power is set to $7.5 \mathrm{dBm}$. There are 10 source/sink data pairs sending UDP data at an application rate of 2.048 $\mathrm{kb} / \mathrm{s}$ each. This is typically done at a rate of 464 -byte packet per second. Application data is started at a random time between 50 and 51 seconds and continues to the end of the simulation.

\section{Performance Comparison Of The Protocols}

Fig. 3 shows the packet delivery ratio of DSDV. Fig. 4 shows the graph between packet received and received rate of AODV. Fig. 5 shows the graph of OLSR for packet delivery ratio. The output results gives us graphs on packet delivery for each protocols. From these three protocols OLSR shows better performance than DSDV and AODV. Although OLSR does not perform at the beginning but after 50 seconds it does well, it sent and received the packets. In DSDV, the packets are dropping. The received rate is very much low or very much high. AODV shows average performance throughout the simulation. AODV shows better results than DSDV. This means DSDV shows worst performance than AODV and OLSR.

\begin{tabular}{|ll|}
\hline simulator & NS-3 \\
Protocols studied & AODV,DSDV,OLSR \\
Simulation time & 600 \\
Simulation area & $300^{*} 1500$ \\
Transmit power & $7.5 \mathrm{dBm}$ \\
Node movement & Random Waypoint \\
model & \\
speed & $20 \mathrm{~m} / \mathrm{s}$ \\
Bandwidth & $2 \mathrm{Mb} / \mathrm{s}$ \\
\hline
\end{tabular}

Table. 1 Simulation parameters 


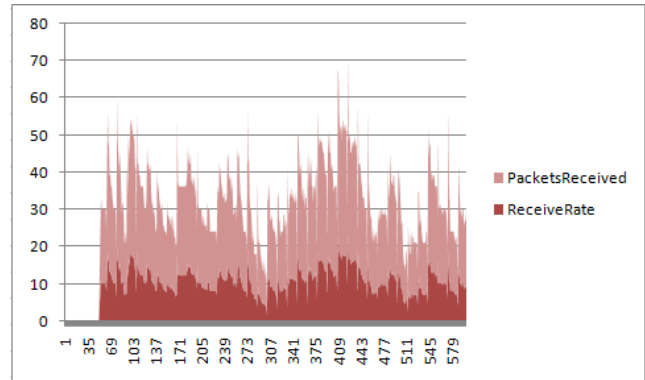

Fig. 3 Packet delivery for DSDV

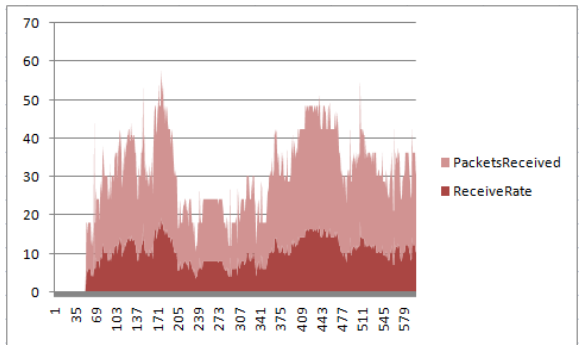

Fig. 4 Packet delivery for AODV

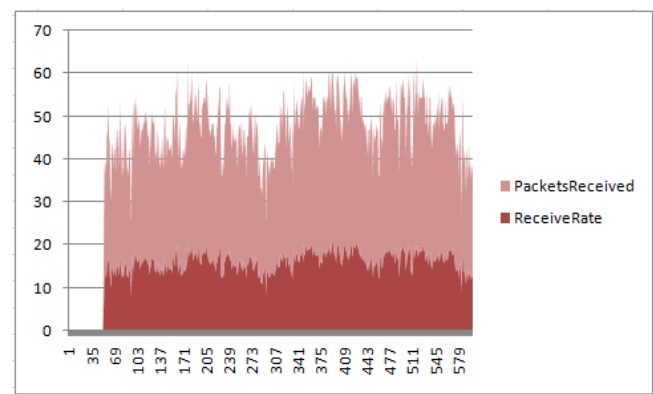

Fig. 5 Packet delivery for OLSR

\section{Conclusion}

In this paper, NS-3 simulator has been used, we evaluated the performance of widely used ad hoc network routing protocols. The simulation characteristics used in this research that is packet delivery. It is very important for performance evaluation of any networking protocol. In this paper we compare three protocols AODV, DSDV, OLSR. We simulated each protocol for adhoc network for 50 nodes. We run simulation for 600 seconds normally the AODV considered to be better on other protocols but here in case of wifi, OLSR shows better performance as compared to DSDVand AODV in context to packet delivery status.

\section{Future Work}

In the future, It is possible to change the mobility and density of the network by directly modifying the speed and the number of nodes. It is also possible to change the characteristics of the network by changing the transmit power (as power increases, the impact of mobility decreases and the effective density increases). Other new protocols performance could be studied.

\section{References}

[1] Kumar Manoj, Parmanand, S.C. Sharma \& S.P. Singh,“ Peformance of QoS Parameter in Wireless Ad hoc Network(IEEE 802.11b)", October 2009.

[2] Priti Garg, Asma Tuteja, "Comparative Performance Analysis of Two Ad-hoc Routing Protocols",International Conference on Network and Electronics Engineering IPCSIT vol.11, 2011.

[3] C.M barushimana, A.Shahrabi, Comparative Study of Reactive and Proactive Routing Protocols Performance in Mobile Ad-Hoc Networks, Workshop on Advance Information Networking and Application, Vol. 2, May, 2003, pp. 679-684,.

[4] C.E.Perkins and E.M.Royer, Ad-Hoc On Demand Distance Vector Routing, Proceedings of the 2nd IEEE Workshop on Mobile Computing Systems and Applications, Feb, 1999, pp.90-100

[5] Md. Golam Kaosar, Hafiz M. Asif, Tarek R. Sheltami, Ashraf S.Hasan Mahmoud, Simulation-Based Comparative Study of On Demand Routing Protocols for MANET, International Conference on Wireless Networking and Mobile Computing, Vol. 1, December 2005, pp. $201-206$.

[6] D. Johnson and D, Maltz, Dynamic source routing in ad hoc wireless networks, 1996. 
[7] Perkins, Charles E. and Bhagwat, Pravin, Highly Dynamic Destination-Sequenced Distance-Vector Routing (DSDV) for Mobile Computers, 1994

[8] T. Clausen and P. Jaqcquet, Optimized Link State Routing (OLSR) Protocol, RFC 3626, IETF Networking Group, October 2003

[9] Jan Schaumann, Analysis of the Zone Routing Protocol December 2002.

[10] Z. J. Hass and M. R. Pearlman, Zone Routing Protocol (ZRP), Internet draft available at www.ietf.org, November 1997.

[11] V. Park and S. Corson, Temporally Ordered Routing Algorithm (TORA) Version 1, Functional specification, IETF Internet draft, December, 1998.

[12] Kuppusamy, P., Thirunavukkarasu, K. and Kalaavathi, B. , A study and comparison of OLSR, AODV and TORA routing protocols in ad hoc networks, 3rd International Conference on Electronics Computer Technology (ICECT), 2011

[13] Anna Hac, "Mobile Telecommunications protocols for data networks", John Wiley \& Sons Ltd, The Atrium, Southern Gate, Chichester,West Sussex PO19 8SQ, England. 